\title{
Article
}

\section{Correcting for interplanetary scattering in velocity dispersion analysis of solar energetic particles}

Laitinen, Timo, Huttunen-Heikinmaa, K, Valtonen, E and Dalla, Silvia

Available at https://clok.uclan.ac.uk/12037/

Laitinen, Timo orcid iconORCID: 0000-0002-7719-7783, Huttunen-Heikinmaa, $K$, Valtonen, E and Dalla, Silvia orcid iconORCID: 0000-0002-7837-5780 (2015) Correcting for interplanetary scattering in velocity dispersion analysis of solar energetic particles. The Astrophysical Journal, 806 (1). ISSN 0004-637X

It is advisable to refer to the publisher's version if you intend to cite from the work. http://doi.dx.org/10:1088/0004-637X/806/1/114

For more information about UCLan's research in this area go to http://www.uclan.ac.uk/researchgroups/ and search for <name of research Group>.

For information about Research generally at UCLan please go to http://www.uclan.ac.uk/research/

All outputs in CLoK are protected by Intellectual Property Rights law, including Copyright law. Copyright, IPR and Moral Rights for the works on this site are retained by the individual authors and/or other copyright owners. Terms and conditions for use of this material are defined in the policies page.

\section{CLoK}

Central Lancashire online Knowledge www.clok.uclan.ac.uk

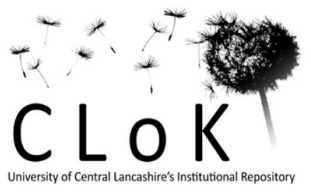




\title{
CORRECTING FOR INTERPLANETARY SCATTERING IN VELOCITY DISPERSION ANALYSIS OF SOLAR ENERGETIC PARTICLES
}

\author{
T. Laitinen ${ }^{1}$, K. Huttunen-HeikinmaA ${ }^{2}$, E. Valtonen ${ }^{2}$, and S. Dalla ${ }^{1}$ \\ ${ }^{1}$ Jeremiah Horrocks Institute, University of Central Lancashire, UK; tlmlaitinen@uclan.ac.uk \\ ${ }^{2}$ Department of Physics, University of Turku, Finland \\ Received 2014 October 7; accepted 2015 April 22; published 2015 June 10
}

\begin{abstract}
To understand the origin of Solar Energetic Particles (SEPs), we must study their injection time relative to other solar eruption manifestations. Traditionally the injection time is determined using the Velocity Dispersion Analysis (VDA) where a linear fit of the observed event onset times at $1 \mathrm{AU}$ to the inverse velocities of SEPs is used to derive the injection time and path length of the first-arriving particles. VDA does not, however, take into account that the particles that produce a statistically observable onset at $1 \mathrm{AU}$ have scattered in the interplanetary space. We use Monte Carlo test particle simulations of energetic protons to study the effect of particle scattering on the observable SEP event onset above pre-event background, and consequently on VDA results. We find that the VDA results are sensitive to the properties of the pre-event and event particle spectra as well as SEP injection and scattering parameters. In particular, a VDA-obtained path length that is close to the nominal Parker spiral length does not imply that the VDA injection time is correct. We study the delay to the observed onset caused by scattering of the particles and derive a simple estimate for the delay time by using the rate of intensity increase at the SEP onset as a parameter. We apply the correction to a magnetically well-connected SEP event of 2000 June 10 , and show it to improve both the path length and injection time estimates, while also increasing the error limits to better reflect the inherent uncertainties of VDA.
\end{abstract}

Key words: diffusion - methods: data analysis - Sun: particle emission - turbulence

\section{INTRODUCTION}

During solar eruptions, charged particles are accelerated up to relativistic energies, to form the solar energetic particle (SEP) population of the cosmic rays observed by in situ instruments at different locations in the heliosphere. The particles are believed to be accelerated in flares and coronal mass ejection (CME)driven shock waves (Reames 1999). However, the relative importance of the flare and CME processes on the origin of the observed SEP populations is still under scientific discussion, and opinions differ on how the eruption phenomena and the SEP production are connected (e.g., Cane et al. 2010; Gopalswamy et al. 2012; Aschwanden 2012).

The difficulty in deducing the particle acceleration scenarios during solar eruptions stems from the nature of SEP observations. The propagation of the charged SEPs is affected by the interplanetary magnetic field. The SEPs are guided by the large-scale Archimedean spiral structure of the interplanetary magnetic field, the Parker Spiral. Solar wind is turbulent, and the particles scatter off the inhomogeneities of the magnetic field (e.g., Parker 1965). Thus, the particle propagation is diffusive rather than direct propagation from the acceleration site to the in-situ particle detectors, typically at 1 AU from the Sun. In order to understand the connections between the components of a solar eruption and the observed SEP intensities, we must understand the propagation of SEPs in the interplanetary space, and deconvolve it from the observations. This has been done for several SEP events (see, e.g., Kallenrode 1993; Torsti et al. 1996; Laitinen et al. 2000; Dröge 2003; Agueda et al. 2009), with most recent works introducing cross-field diffusion into the modeling of SEP events (e.g., Zhang et al. 2009; Dröge et al. 2010; He et al. 2011; Dresing et al. 2012).

Deconvolving of the interplanetary transport from the in-situ SEP observations is, however, not simple, and is usually performed only in case studies. For larger statistical studies, simpler methods to obtain the injection time of the SEPs are commonly used. The popular choice is to use the Velocity Dispersion Analysis (VDA), where the first-observed particles are assumed to have propagated without scattering (e.g., Lin et al. 1981; Reames et al. 1985; Torsti et al. 1998; Krucker \& Lin 2000; Tylka et al. 2003; Reames 2009; Vainio et al. 2013). The possible uncertainties on the arrival times of first particles caused by interplanetary transport effects are typically not evaluated in these studies.

The validity of the VDA method has been studied by using numerical SEP simulations that solve the focused transport equation describing particle propagation in interplanetary space. By using onset times at $1 \mathrm{AU}$ obtained from simulated SEP time-intensity profiles at different energies, it has been shown that for strong scattering conditions the VDA can result in large errors for the injection times and path lengths of the particles (Kallenrode \& Wibberenz 1990; Lintunen \& Vainio 2004; Sáiz et al. 2005). These studies, however, defined the onset time relative to the maximum intensity (e.g., the time when the intensity reaches $1 \%$ of the maximum intensity), which is not the common practice when analyzing real SEP events. Unlike in simulated SEP events, real events may have a complex structure, due to local interplanetary magnetic field structures and multiple, energy-dependent injection components (e.g., Laitinen et al. 2000). This may affect the time profile of the event before the maximum intensity is reached. Thus, to get the best estimate of the beginning of the injection, the onset of an SEP event is typically determined by using the moment when the SEP intensity exceeds the pre-event background by a statistically significant amount (e.g., Huttunen-Heikinmaa et al. 2005). Laitinen et al. (2010) analyzed simulated SEP events with VDA by adding a pre-event background to the time-intensity evolution of the SEP event, 
and using the background-exceeding time as the event onset time. They found that the errors in VDA have strong dependence on the pre-event and event maximum spectra.

In this work, we build on the results of Laitinen et al. (2010) and discuss the complexity of the effect of the pre-event background and the role of scattering in different types of SEP events, and show how different event types can result in very different VDA results. We study the SEP time-intensity profile of energetic protons at the time of the observed onset, and derive a simple estimation for the delay the particles experience due to the scattering in interplanetary space. We show that this delay estimate can successfully be used as a correction to the observed onset times to improve the accuracy of the VDA. We apply the correction and its error limits to simulated SEP events and the SEP event of 2000 June 10, and show that the method improves the deduced injection time in both cases.

To clarify the used terminology, we use the term "injection time" to describe the time of release of SEPs at or near the Sun, "onset time" to describe the time when the SEP intensities are observed to rise at $1 \mathrm{AU}$ (see the discussion below), and "launch time" as the time when a CME is estimated to lift off. Furthermore, the symbol $t_{i}$ refers to the injection time obtained by VDA, and $t_{o}$, the onset time obtained from the simulated events. The time required for a scatter-free particle with velocity $v$ to propagate distance $s$, i.e., $s / v$, is referred to as "scatter-free time."

\section{MODELS}

\subsection{Solar Particle Event Modeling}

In this work, we study the effect of pre-event background on SEP injection times as determined by the VDA method, in the presence of interplanetary scattering and different injection profiles. We solve the focused transport equation of energetic protons,

$$
\begin{aligned}
\frac{\partial f}{\partial t} & +\frac{\partial}{\partial z} v \mu f+\frac{\partial}{\partial \mu} \frac{v}{2 L}\left(1-\mu^{2}\right) f \\
& -\frac{\partial}{\partial \mu}\left(1-\mu^{2}\right) \nu \frac{\partial f}{\partial \mu}=Q(z, \mu, t),
\end{aligned}
$$

with $f(v, \mu, z, t)$ the particle distribution function, $v, \mu, z$, and $t$ the speed, pitch angle cosine, position along field line and time, respectively, $L$ the focusing length, $\nu$ the scattering frequency, and $Q$ describing the particle source. The equation is solved using Monte Carlo simulations, where test-particles are propagated and focused along the Parker spiral in a frame corotating with the Sun, and scattered isotropically in pitch angle, with $\nu=v /(2 \lambda)$, where $\lambda$ is the parallel scattering mean free path (e.g., Torsti et al. 1996; Kocharov et al. 1998). Adiabatic deceleration is taken into account by scattering the particles in the co-rotating solar wind frame. Propagation of paricles across the Parker spiral direction is not considered.

We use the initial energy range from 1 to $120 \mathrm{MeV}$ with a power-law spectrum $\propto E^{-3}$. This power law is used for all runs, to reduce the parameter space. The particles are injected at $0.01 \mathrm{AU}$, at time $t=0$, with a reflecting boundary at the Sun, and followed for $48 \mathrm{hr}$. The Parker spiral is parametrized by a constant solar wind velocity of $400 \mathrm{~km} \mathrm{~s}^{-1}$ and solar rotation period of 25.35 days, resulting in Parker spiral length of 1.17 AU. We use a constant radial mean free path, with values $\lambda_{r}=0.3 \mathrm{AU}$ at $1 \mathrm{GV}$ rigidity to represent moderate scattering conditions, and $1 \mathrm{AU}$ at $1 \mathrm{GV}$ rigidity to represent weak scattering conditions (e.g., Palmer 1982). The mean free path is taken to depend on the particle rigidity, as $R^{1 / 3}$, consistent with quasilinear theory for Kolmogorov slab spectrum (Jokipii 1966) and observations (e.g., Dröge 2000).

The Monte Carlo simulations are used to obtain a response, as observed at $1 \mathrm{AU}$, for an impulsive injection of protons, at 20 logarithmically spaced energy channels between 1 and $100 \mathrm{MeV}$. The upper limit of the channels is chosen to be $100 \mathrm{MeV}$ rather than $120 \mathrm{MeV}$, because particles experience adiabatic deceleration. In order to mimic more realistic particle release scenarios, we convolve the impulsive responses with two injection profiles at the Sun: a fast injection model, with 30 minutes of linear increase of injection strength, followed by 570 minutes of linear decay; and a slow injection model, with 570 minutes of linear increase, followed with 30 minutes of linear decay. These profiles were selected because CME-related SEP acceleration has been estimated to be most efficient when the $\mathrm{CME}$ is at $5-15 R_{\odot}$ (e.g., Kahler 1994). A CME with velocity $2000 \mathrm{~km} \mathrm{~s}^{-1}$ reaches $5 R_{\odot}$ in half an hour, corresponding to our fast injection model. The slow injection model accounts for slower CMEs, higher maximum injection heights, poor connection between the spacecraft and acceleration region, and possible cross-field transport of SEPs, that may cause a gradual injection of particles.

We define our study parameters using SEP intensities that can be observed directly at $1 \mathrm{AU}$. We scale the convolved time-intensity profiles of simulated SEPs at $1 \mathrm{AU}$ so that the spectrum calculated from the maxima of the scaled intensities at each channel forms a power law,

$$
I_{\mathrm{ev}}=I_{0 \mathrm{ev}}\left(\frac{E}{E_{0}}\right)^{-\alpha_{\mathrm{ev}}},
$$

where $I_{0 \mathrm{ev}}$ is the SEP event maximum intensity at energy $E_{0}=88 \mathrm{MeV}$, and $\alpha_{\mathrm{ev}}$ the power-law index of the spectrum. The resulting event time-intensity profiles are overlaid on a constant pre-event background, which also follows a power-law energy spectrum,

$$
I_{\mathrm{bg}}=I_{0 \mathrm{bg}}\left(\frac{E}{E_{0}}\right)^{-\alpha_{\mathrm{bg}}},
$$

where $I_{0 \mathrm{bg}}$ is the background intensity at $E_{0}$ and $\alpha_{\mathrm{bg}}$ the background power-law index. An example of the spectra is shown in Figure 1.

\subsection{Velocity Dispersion Analysis}

The VDA is based on the assumptions that the first particles observed at a given distance from the Sun have been released simultaneously, propagate the same path length, and experience no scattering or energy changes. Under these conditions, the arrival time, $t_{o}$, of the particles to the observer at distance $s$ along the magnetic field line is given by

$$
t_{o}(v)=t_{i}+\frac{s}{v},
$$

where $t_{i}$ is the particles' injection time at the Sun, $s$ the traveled distance and $v$ the particle velocity. Thus, knowing the observed onset times at $1 \mathrm{AU}$, and the velocities of the particles, a simple linear fitting of this data according to the 


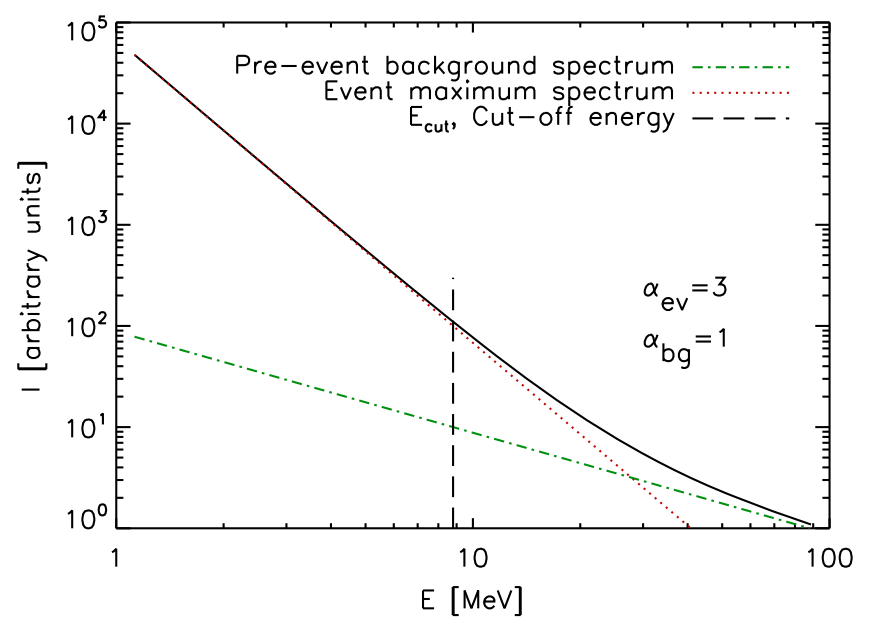

Figure 1. Example of the event maximum spectrum (red dotted curve), the preevent background spectrum (green dashed-dotted curve), with the total intensity spectrum at event maximum given by solid black curve. The dashed vertical black line depicts the energy $E_{\text {cut }}$, where the event maximum intensity is an order of magnitude times the background intensity. The event maximum spectrum power-law index is 3 , and the background power-law index 1 .

Equation (4) gives the particles' injection time at the Sun, and the path length traveled by the particles. In the simulated events, the injection of the particles starts at $t=0$, thus a successful VDA fit would give $t_{i}=0$ and $s=1.17$ AU.

While all of the VDA assumptions can be questioned, in this study we concentrate only on the effect of the scattering on the derived injection time and path length. In particular, we will study the common practice of defining SEP onset as the moment the intensity is discernible from the pre-event background, and how this practice affects the VDA results.

The SEP event onset time, observed by particle detectors, is often difficult to determine due to the low counting rate of particles of the ambient energetic particle population before and at the very beginning of the event. To determine the onset time, typically a threshold of one or several standard deviations above the pre-event background is used to define the onset in a statistically significant way (see also Huttunen-Heikinmaa et al. 2005, for an alternative method). This results in a delay for the observed onset. In order to mimic this effect, we define the onset to be observed when the intensity at 1 AU rises $10 \%$ above the pre-event background.

\section{RESULTS}

\subsection{Parametric Study}

We show an example of an analyzed event in Figures 2 and 3. In Figure 2, we show the time evolution of a simulated event, with maximum intensity spectrum $\propto E^{-3}$, taking place when the pre-event background spectrum is proportional to $E^{-1}$, with the event intensity an order of magnitude below the background intensity at energy $88 \mathrm{MeV}$, and $\lambda_{r}=0.3 \mathrm{AU}$, and fast injection. The onset times of the intensity increases are shown by symbols, with the brown thick curve crossing the timeintensity curves on the left showing the theoretical time of arrival of particles in the case of particles following the assumed 1.17 AU Parker Spiral field without scattering. As can be seen, the intensity increase begins significantly later than the scatter-free time at all energy channels.
The effect of the delay on the VDA can be seen in Figure 3, where we show the onset time plotted against $1 / \beta$ (the red and green symbols). As can be seen, the onset times are clearly delayed from the velocity dispersion pattern for particles that are observed immediately after scatter-free propagation to 1.17 AU (dashed blue line). In addition, not all observed onsets follow the linear velocity dispersion pattern. In our example event, at large energies the arrival time dependence on $1 / \beta$ is inverted, with onsets at higher energeies observed later than at lower energies, due to the pre-event background. Such energy channels clearly must be excluded from the velocity dispersion fit. In order to achieve this, we have excluded energy channels where $I_{\mathrm{ev}}(E) / I_{\mathrm{bg}}(E)<10$ (the green curves and small symbols in Figures 2 and 3 ).

However, even after removing the nonlinearly behaving energy channels from the velocity dispersion fit, the pre-event background and scattering still have a substantial effect on the determination of the SEP injection time. The observed onset time at different energy channels is delayed by 20-40 minutes from the scatter-free arrival time. The VDA-fitted injection time for this event is 21 minutes later than the actual release time of particles at the Sun. The path length, 1.26 AU, is clearly longer than the nominal 1.17 AU Parker spiral length in our model. The increased path length is due to the low-energy (high $1 / \beta$ ) particles having a longer delay relative to the scatterfree arrival time (blue dashed line in Figure 3), as compared to the higher-energy particles.

In Figure 4, we show the VDA fits for $\lambda_{r}=0.3 \mathrm{AU}$ for the fast (left column) and slow (right column) injection model, and different types of events. In the top panels, we show an SEP event with the maximum spectrum softer than pre-event background spectrum. This type of event may be observed when the pre-event background is not affected by preceding SEP events (e.g., Valtonen et al. 2001). In such an event, the intensities at higher energies are masked more efficiently by the pre-event background than at lower energies. As can be seen, the VDA path lengths are typically close to or slightly longer than the nominal Parker spiral length, with the obtained injection time considerably later than the actual solar injection time.

In the event shown in the middle panels, the pre-event and event maximum spectral indices are equal. This can take place for example when a soft-spectrum SEP event follows a harderspectrum SEP event. The delay with respect to the scatter-free arrival time is significantly larger at low energies. This results in a significantly longer path length, of $1.52 \mathrm{AU}$ in the case of the fast injection model (left middle panel), and 1.75 AU for the slow injection model (right middle panel). The long path length, however, compensates partly the delay caused by scattering, resulting in only a 2 minute error for the injection time determination with the fast injection model.

The bottom panels of Figure 4 depict an event where a hard SEP event takes place on a soft pre-event background. This typically takes place when a previous SEP event is still decaying at the time of a new SEP event. In this case, the lower energies are efficiently masked by the pre-event background, and the velocity dispersion fit results in a very long path length. As can be seen in both the fast and slow injection models (bottom left and right panels, respectively), the obtained injection time can precede the real injection time in such cases. 


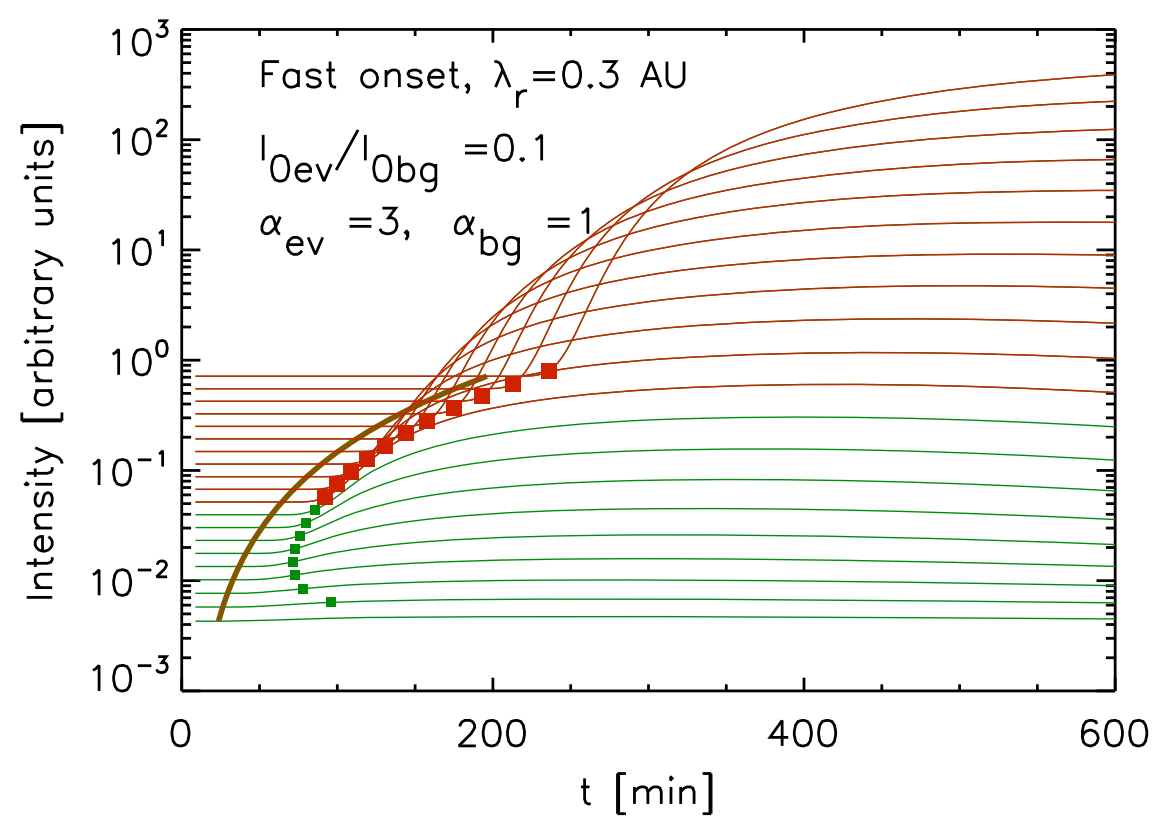

Figure 2. Example of a proton event with energies $1-100 \mathrm{MeV}$ overlaid on a pre-event background. The lowest-energy intensities are on the top of the figure, the highest on the bottom. The onset times, as defined in the text, are marked by squares. The red curves and the large red squares represent the channels where the event maximum intensity is over 10 times the background, the green curves and the small green squares represent the smaller-intensity energies. The thick brown curve represents the scatter-free arrival time of particles.

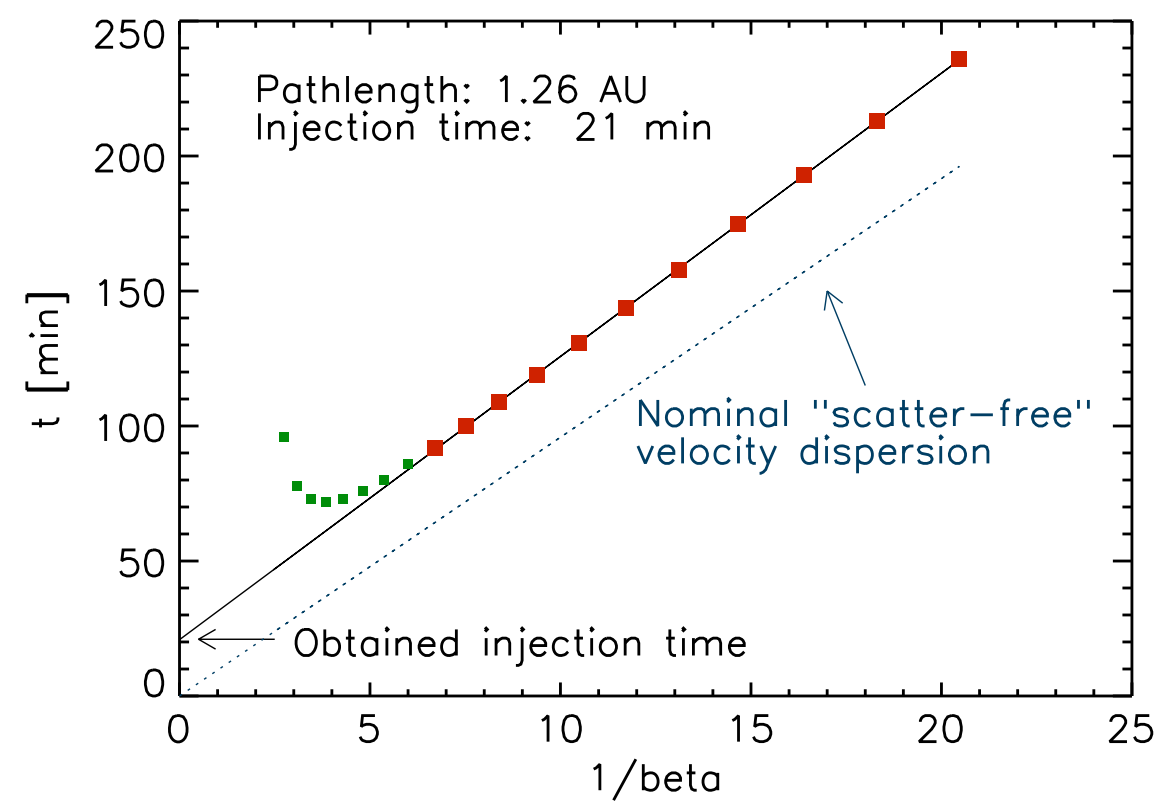

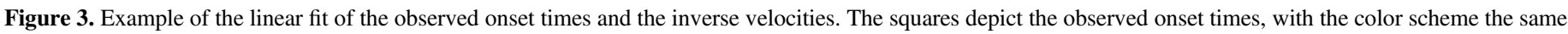

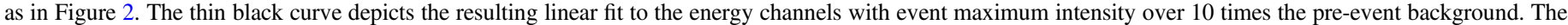

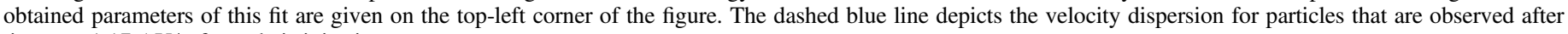
time $t=1.17 \mathrm{AU} / v$ from their injection.

Figure 4 also shows that the injection profile of the particles has a significant effect on the VDA results, with the comparison of fast and slow injection profiles on the left and right columns, respectively. As can be seen, the effect of the pre-event and event maximum spectral shapes on the velocity dispersion pattern is similar for both injection models. However, the delay times for individual energy channels are longer in the slow injection case, and the resulting error in injection time is also larger.

As shown in Figure 4, the assumption of scatter-free propagation can result in significant systematic errors for the
SEP injection time and the traversed path length. This error is caused by the delay of particles due to interplanetary scattering, which should be taken into account in the VDA as

$$
t_{o}(v)=t_{i}+\frac{s}{v}+t_{d}\left(v, \lambda, t_{o}\right)
$$

The estimation of the delay time $t_{d}$ is not trivial, as it depends on scattering conditions, injection profile, and the level of the pre-event background relative to the SEP event intensity. Also the estimation of the resulting error in VDA is not straigthforward: as shown in the middle panels of Figure 4, an 

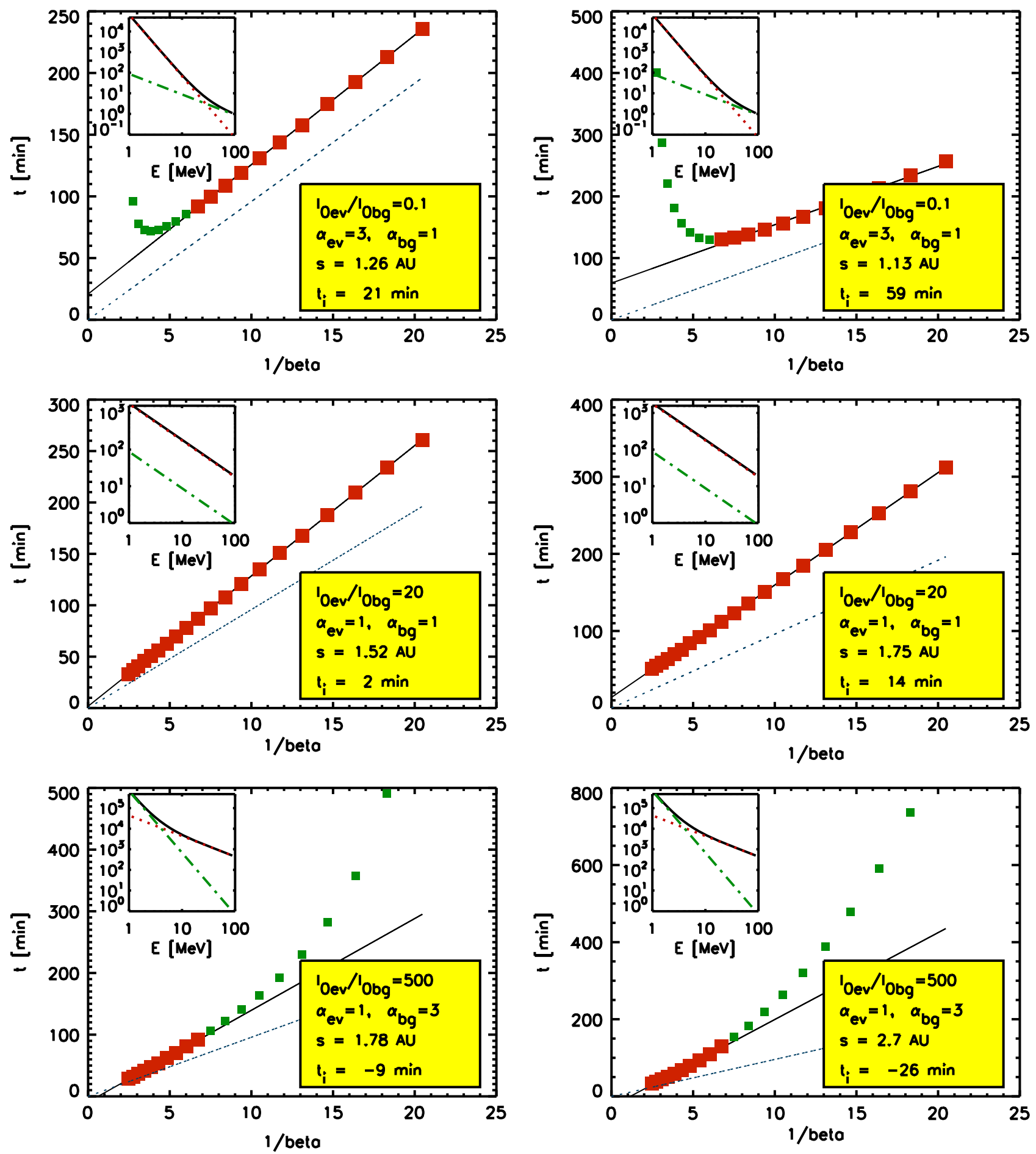

Figure 4. Onset times fitted for simulated events with different event maximum and background spectra, and injection profiles. The left column presents the results of the fast injection model, and the right the slow injection model, with $\lambda_{r}=0.3 \mathrm{AU}$. The symbols represent the obtained onset times at different energies, with the large (small) symbols for channels where the intensity at event maximum is more (less) than an order of magnitude above the pre-event background. The solid curve shows the $1 / \beta$-fit to the onsets, and the dashed curve the fit expected for scatter-free particles. The inset in the top left of the panels shows the event maximum spectrum (dashed curve), background spectrum (dotted-dashed curve) and the total spectrum (solid curve).

error in the path length can compensate the error in the injection time determination. Such a complicated relation between the observed velocity dispersion and the injection is difficult to analyze, and may skew the results of large statistical SEP studies, where fitting of the transport of SEPs is not feasible.

\subsection{Analysis of the Delay Time in VDA}

The rise time of SEP intensities above the pre-event background is not the only observable of an SEP event onset. As shown in Figure 2, the intensities rise initially roughly exponentially, with different rise rates at different energies and 


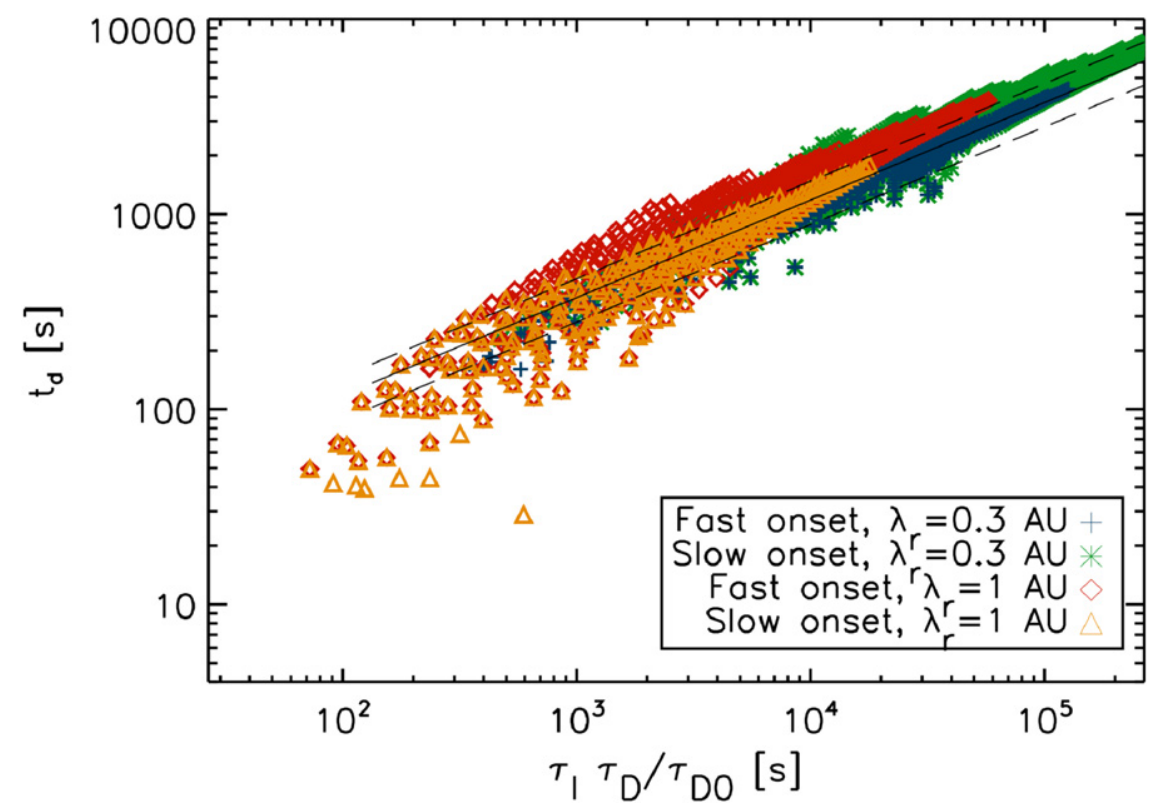

Figure 5. Scatter plot of $t_{d}=t_{i}-s / v$ against the scaled intensity increase scale time, for simulations with different mean free paths and injection profiles. The solid curve represents the best fit of the Equation (10) to the simulation data, with the dashed curves depicting the error limits of the fit.

intensities. This gradual rise, as opposed to an immediate rise to the maximum intensity, is a result of the particle scattering in interplanetary space. In the following, we study the rate of intensity increase at the time of SEP event onset.

For a diffusion process with scattering mean free path $\lambda$, the intensity of particles with velocity $v$ at distance $r$ and time $t$ is given as

$$
I_{\mathrm{diff}}(r, t) \propto \frac{1}{(4 \lambda v t / 3)^{3 / 2}} \exp \left\{-\frac{3 r^{2}}{4 \lambda v t}\right\} .
$$

From this, we can obtain the timescale of the intensity increase as

$$
\tau_{I}^{-1} \equiv \frac{1}{I_{\mathrm{diff}}} \frac{d I_{\mathrm{diff}}}{d t}=\frac{3}{2} \frac{1}{t}\left(\frac{1}{2} \frac{r}{\lambda} \frac{r}{v t}-1\right) .
$$

The diffusion approach is valid only for times $t \gg \lambda / 3 v$ and $t \gg r / v$. Indeed, the intensity maximum takes place at $t_{\max }=r /(2 \lambda) r / v$, which for $\lambda>r / 2$ is before the particles with velocity $v$ can arrive scatter-free to distance $r$, clearly an unphysical result. However, as there are no analytic descriptions for the initial phase for diffusively spreading particles, we will use diffusion as a starting point of our study.

We are only interested in the initial increase of the SEP event, and use only times when the intensity is less than $I_{\mathrm{ev}}(E) / 10$. Thus we only use the first term in Equation (7). Rewriting $\tau_{I}$ using the diffusion timescale, $\tau_{D}=3 r^{2} /(2 \lambda v)$, we arrive with a simple scaling between the time from SEP injection at the Sun, $t$, and the intensity increase timescale $\tau_{I}$ as

$$
\frac{1}{2} \tau_{I} \tau_{D}=t^{2}
$$

To study this scaling, we simulated a large number of SEP events with the ratio $I_{0 \mathrm{ev}} / I_{0 \mathrm{bg}}$ ranging from $10^{-4}$ to $10^{4}$, and the difference of the event maximum and pre-event background spectral indices from -3 to 3 , both fast and slow injection models, and moderate and weak scattering conditions. The intensity increase timescale was obtained from the simulated events by using intensities at two consecutive times, with

$$
\tau_{I}^{-1}=\frac{\ln \left(I_{\mathrm{ev}}\left(t_{2}\right) / I_{\mathrm{ev}}\left(t_{1}\right)\right)}{t_{2}-t_{1}} .
$$

The scaling in Equation (8) is obtained by multiplying the intensity increase timescale by $\tau_{D} / \tau_{D 0}$. As the radial mean free path $\lambda_{r}$ is constant in our model, we use the $\lambda_{r}$ and $r=1 \mathrm{AU}$ in the diffusion timescale. The reference value, $\tau_{D 0}$ is calculated using $\lambda_{r}=1 \mathrm{AU}$ and $v=c$.

We found that comparing $\tau_{I}$ with the onset time did not result in the expected power-law behavior given by Equation (8), due to the inability of the diffusion description to describe the early time evolution of SEP propagation. However, as shown in Figure 5, the power-law behavior is retained when plotting $\tau_{I} \tau_{D} / \tau_{D 0}$ as a function of delay time $t_{d}$, for several orders of magnitude, for different particle energies, scattering conditions and injection profiles. The delay time is reminescent of the concept of signal speed in modeling the particle propagation with the Telegraph equation (Fisk \& Axford 1969). However, as shown by Effenberger \& Litvinenko (2014), the Telegraph equation models the initial phase of an SEP event poorly, and is not hence addressed further in this study.

As seen in Figure 5, the power law suggested by Equation (8) is not exact. Thus, to estimate the delay time for using it as a correction for VDA, as in Equation (5), we fitted the data in Figure 5 to

$$
t_{d}=a \sqrt{\frac{\tau_{I} \tau_{D}}{2}}
$$

which gave $a=0.61$, with standard deviation $\sigma_{a}=0.15$. This estimate can be used further in correcting for the effects of interplanetary transport on VDA. At larger values of $\tau_{I}$, the diffusive profile is approached and values of $a$ up to unity could be used. 

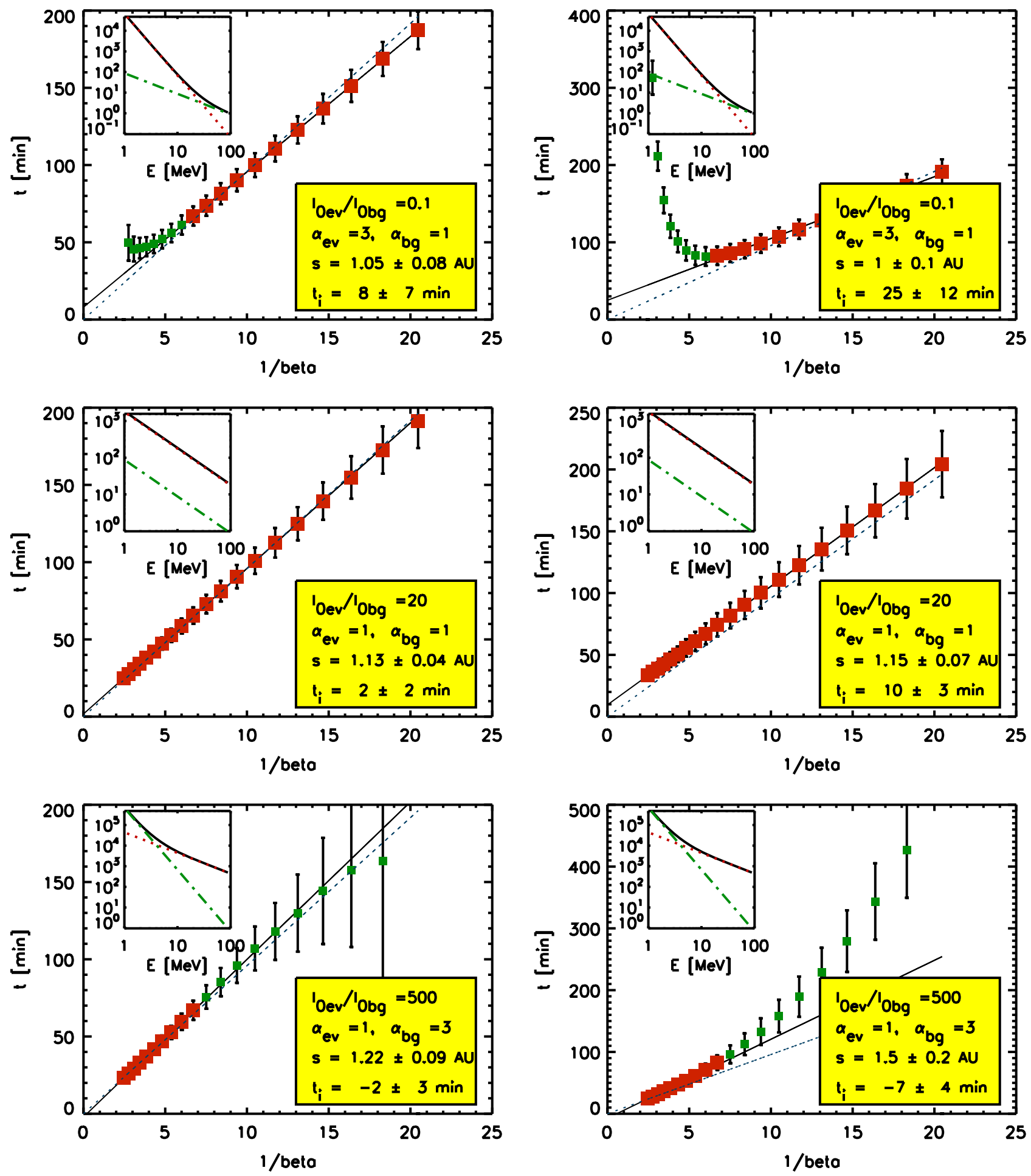

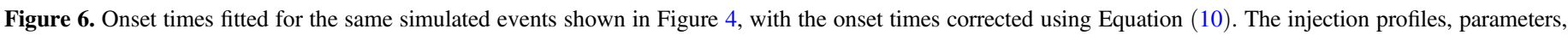
symbols, and curves are the same as in Figure 4.

The data can also be fitted as a power law, resulting in a power-law index of 0.56 , instead of the form suggested by Equation (8). However, we consider the fit to Equation (10) to be a better choice, as it is based on the physics of particle propagation at the time-asymptotic limit, and as such is more likely applicable to other energy ranges and particle species.
We have applied the correction to the observed onset times given by Equation (10) to the six simulated event examples shown in Figure 4, and present the resulting, corrected VDA fits in Figure 6 . The delay time $t_{d}$ given by Equation (10) has been subtracted from the onset times, $t_{o}$, and the error limits as defined by $\sigma_{a}$ have been used in the fitting to obtain error limits for the injection time and the path length. As can be seen, the 


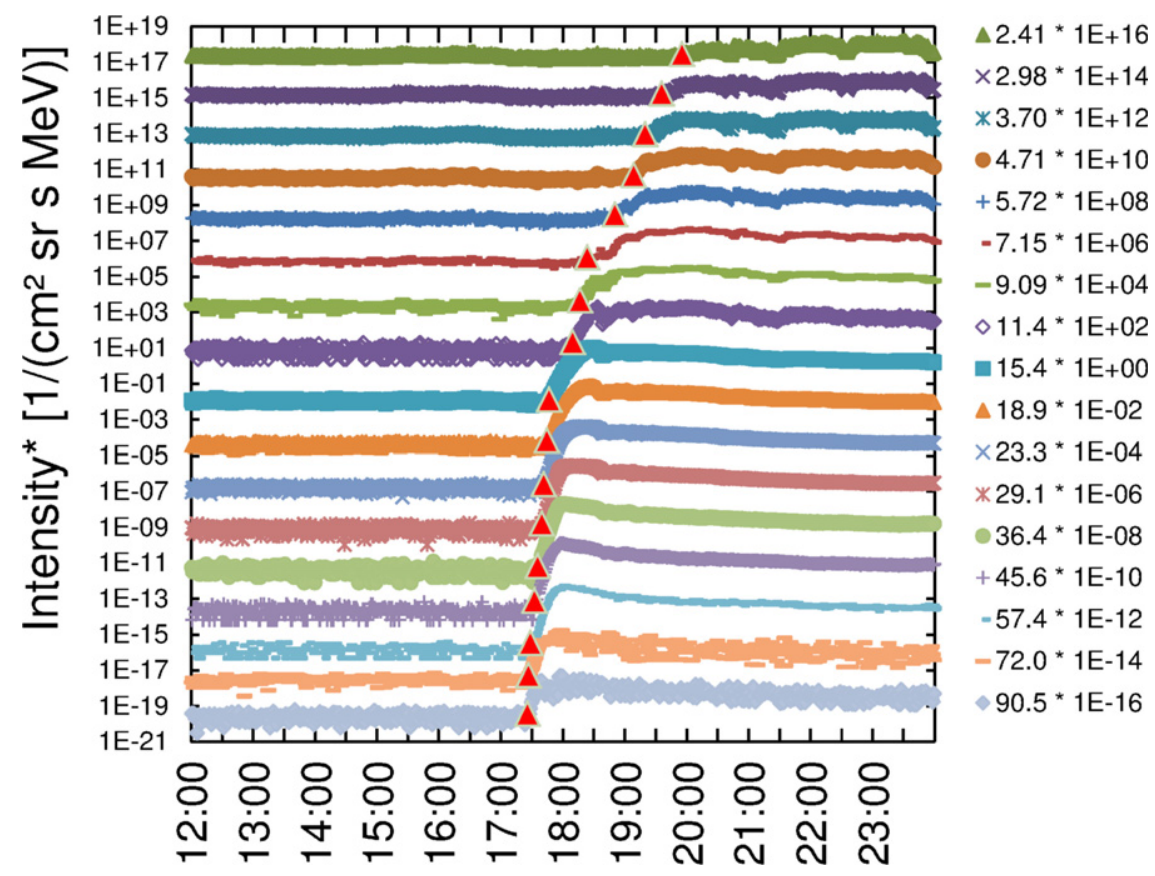

Figure 7. Proton intensities at energies $2.41-90.5 \mathrm{MeV}$ on 2000 June 10 , as observed by the ERNE instrument on board the SOHO spacecraft. The onset times, as determined by the method described in Huttunen-Heikinmaa et al. (2005), are shown with red triangles. The intensities in each consecutive energy channel are multiplied by a factor of 100 to better distinguish onset times.

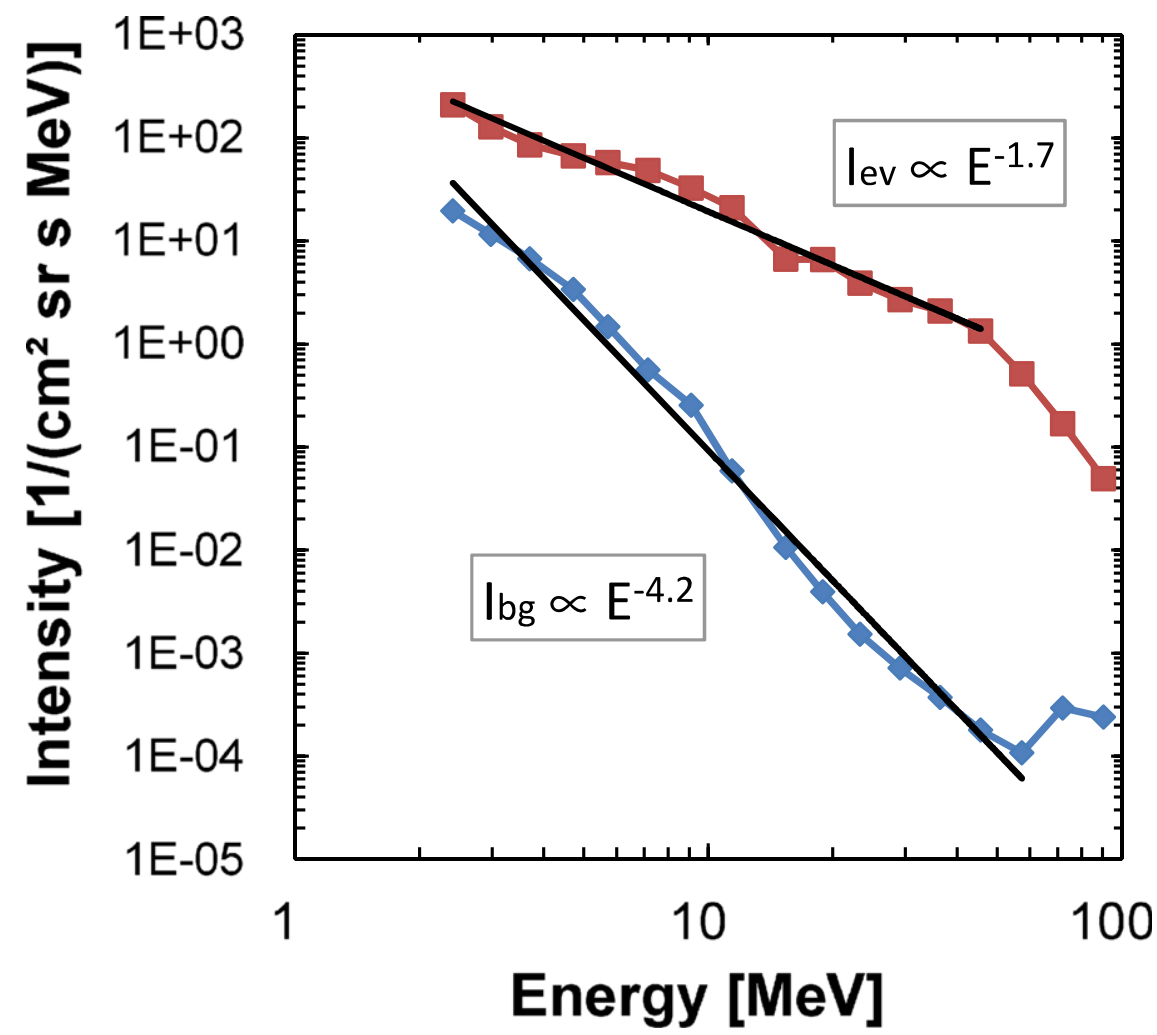

Figure 8. Event maximum (red curve and squares) and pre-event background (blue curve and diamonds) spectra on 2000 June 10, with respective power-law fits.

correction improves both the injection time and the path length estimates considerably. The injection times are correct within the error limits for the fast injection profile (left column), with the path lengths also better reflecting the Parker Spiral length of $1.17 \mathrm{AU}$.
When analyzing real SEP events, the limited counting statistics of the particle detectors cause an uncertainty factor for the delay time through the dependence of $\tau_{I}$ on intensity. We assume that the pre-event background intensity can be evaluated from sufficiently long time period so that its 


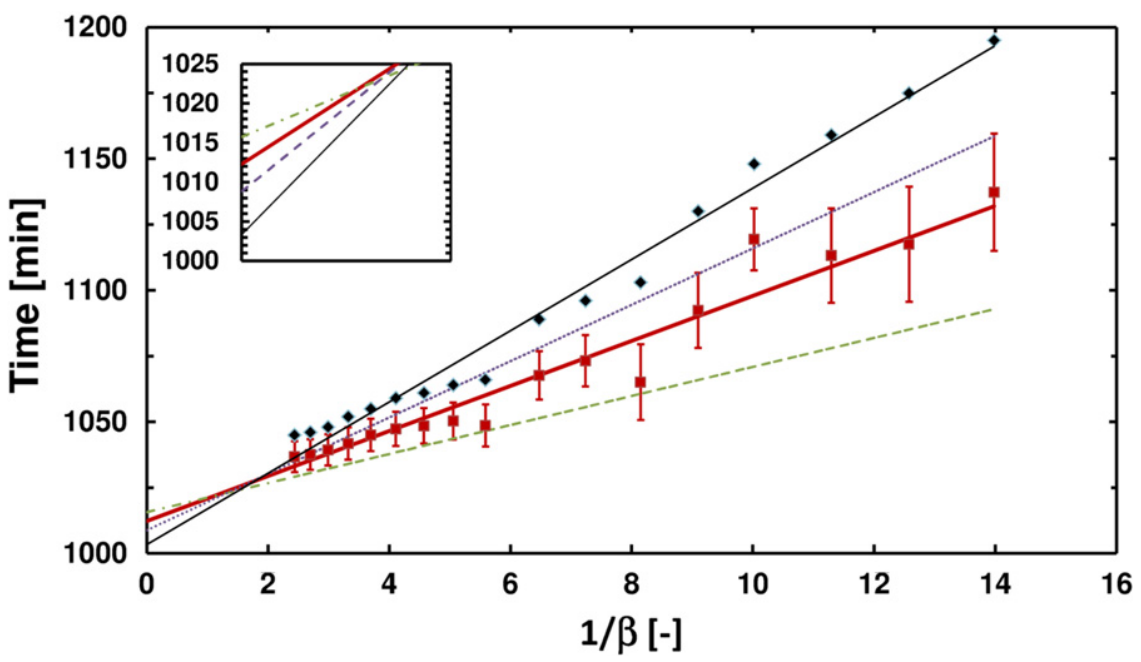

Figure 9. Onset time and velocity dispersion fit correction illustration for the 2000 June 10, event. Observed onset times are represented by black diamonds, and the thin black line is the corresponding least-squares linear fit $\left(s=1.63 \pm 0.06 \mathrm{AU} ; t_{0}=16: 43 \pm 4\right.$ minutes). Red squares represent the corrected onset times using reference mean free path $0.3 \mathrm{AU}$. See the text for details of the error bars. Blue dotted, thick red, and green dashed-dotted lines correspond to weighted least-squares linear fits for corrected onset times of reference mean free paths 1.0, 0.3, and $0.1 \mathrm{AU}$, respectively. These three corrected velocity dispersion fits can be incorporated to corrected values of $s=1.03_{-0.28}^{+0.34} \mathrm{AU}$, and $t_{0}=16: 52_{-8}^{+7}$ minutes . Eight minutes need to be added to the times to make them comparable to electromagnetic observations.

statistical error is insignificant compared to those of intensities at times $t_{1}$ and $t_{2}$ in Equation (9). With this assumption, the statistical error of $\tau_{I}$ can be evaluated as

$$
\frac{\Delta \tau_{I}^{2}}{\tau_{I}^{2}} \approx \frac{1}{\left[\ln \left(I_{2} / I_{1}\right)\right]^{2}}\left(\frac{1}{N_{1}}+\frac{1}{N_{2}}\right),
$$

where the variables $N_{1}$ and $N_{2}$ represent the number of particles detected by the instrument at the two times used to calculate $\tau_{I}$. Using this approximation, we have

$$
\frac{\Delta t_{d}}{t_{d}} \approx \sqrt{\left(\frac{\sigma_{a}}{a}\right)^{2}+\frac{1}{4} \frac{N_{1}+N_{2}}{N_{1} N_{2}\left[\ln \left(I_{2} / I_{1}\right)\right]^{2}} .}
$$

If the relative error from the background determination is significant, it should be included in the error analysis.

In addition to the finite counting statistics, there is still one significant source of uncertainty in Equation (10). The diffusion timescale, $\tau_{D}$, depends on the scattering mean free path of the particles, $\lambda$. While $\lambda$ depends on the amplitude of interplanetary turbulence, it is difficult to estimate, and is typically obtained as a side product of SEP transport fitting. According to several studies (e.g., Palmer 1982), the mean free path varies from 0.08 to $0.3 \mathrm{AU}$, with some recently analyzed events showing significantly longer mean free paths (e.g., Torsti et al. 2004). There is no reason to expect the mean free paths to be normally distributed, thus we evaluate its effect on the delay time estimation by using extreme mean free path values, e.g., 0.1 and 1.0 AU, and obtaining the smallest and largest parameter values (taking also the statistical errors into consideration).

\subsection{June 10 SEP Event}

In order to study the effects of the delay due to scattering, and the ability of the correction derived above to improve the VDA results, we apply the correction to an observed SEP event. In Figures 7 and 8, we show the time-intensity profiles and spectra of the SEP event of 2000 June 10, as observed by ERNE instrument on board the Solar and Heliospheric
Observatory (SOHO) spacecraft (Torsti et al. 1995). The event takes place during a decay phase of an earlier SEP event. For this reason, the pre-event spectrum is very soft compared to the event maximum spectral index, as shown in Figure 8. Thus, this event corresponds to the simulated events in the bottom row of Figure 4.

The SEP event coincides with an M5.2 solar flare and a western halo CME. The flare, located at heliographic coordinates N22 W38, started at 16:40 UT and reached its maximum at 17:02 UT. The CME was launched at 16:45 UT, as given by a linear extrapolation of $\mathrm{SOHO/LASCO}$ observations to solar surface in the CDAW SOHO/LASCO CME list (Gopalswamy et al. 2009). The western location of the eruption implies a good magnetic connection for the SEPs along the Parker spiral to the near-Earth spacecraft, with negligible crossfield propagation effects on the first-observed particles.

We used the VDA method to determine the solar injection time and path length of the $2.41-90.5 \mathrm{MeV}$ protons in this event. Using uncorrected onset times, VDA gave for the solar injection 16:43 \pm 4 minutes solar time. Taking into account the 8 minutes light needs to travel from the Sun to Earth, the particles are injected 11 minutes before the flare maximum time, with the CME estimated to be low in the corona. The path length of the particles obtained from uncorrected onset times is very long, $s=1.63 \pm 0.06 \mathrm{AU}$. This is consistent with the simulated events (bottom row of Figure 4): an event with maximum spectrum harder than the pre-event spectrum will show a very long path length.

We then applied the correction, as given by Equation (10), to the onset times. We show the effect of the correction in Figure 9, where the black diamonds and the black line correspond to the uncorrected onset times and the corresponding VDA fit. The red squares show the corrected onset times, with the associated errorbars, for mean free path $\lambda=0.3 \mathrm{AU}$. We calculated the corrected VDA results using three different mean free paths, depicted with the blue dotted, solid red, and green dashed-dotted curves in Figure 9. As can be seen in the inset of the figure, all three fits give a later time of SEP injection, as compared to the VDA fit without time correction. 
Using the errors given by Equation (11) in the velocity dispersion fit, we obtain error limits for each of the fits with different $\lambda$ values. Using injection time, path length, and the fitting error values we find the corrected injection time as 16: $52_{-8 \text { minutes }}^{+7}+8$ minutes, with path length $s=1.03_{-0.28}^{+0.34}$ AU. Thus, the path length after the correction is consistent with the expected Parker spiral length. The solar injection time, of 16:59 UT corrected for electromagnetic observations, coincides well with the maximum time of the X-ray flare. The CME was observed at 17:08 UT at $2.76 R_{\odot}$. Thus, within the error limits of the injection time, both the flare and CME observations are consistent with potential energetic particle production.

\section{DISCUSSION}

As this study shows, the results of VDA fitting should be used carefully. SEPs scatter in the interplanetary medium, and while the first particles related to an event may indeed be scatter-free, their intensity is likely too low for statistically reliable observation. It should be noted that having a very low level of pre-event counting rates, such as in the case of heavy elements, does not imply that the first observed SEP event particles are scatter-free. The instrument's detection threshold has a similar effect to the observed event onset as the pre-event background intensity level.

It is important to notice that a reasonable path length does not imply a good estimate for the solar injection time. This can be seen in the fits presented in Figure 4: if the path length is nominal, as in the top panels, all of the fitted energies will have almost equal delay time $t_{d}$, which results in a large error for the injection time. If, on the other hand, the path length is long, the delay time is shorter at higher energies (smaller $1 / \beta)$, and the VDA-fitted curve converges toward the scatter-free VDA pattern (middle panels of Figure 4) at the limit of $1 / \beta=0$, and the resulting error in the VDA injection time is small. This can take place in particular when the spectral indices of the preevent background and event maximum spectra are similar. In this case the ratio of maximum and background intensities is independent of energy, and the VDA result is similar to the method used by Lintunen \& Vainio (2004) and Sáiz et al. (2005), who determine the onset time as the time when a fixed fraction of maximum intensity at the energy channel is reached.

It should also be remembered that factors other than the parallel scattering may influence the particle propagation in the interplanetary space. Recent multi-spacecraft analyses of SEP events suggest that SEP events have a wide extent in heliographic longitudes (Dresing et al. 2012, 2014; Wiedenbeck et al. 2013; Richardson et al. 2014). While the large longitudinal spread of particles may be caused by processes low in the corona (see, e.g., Wiedenbeck et al. 2013, for discussion), the observed longitudinal dependence of anisotropy suggests that significant interplanetary cross-field transport is taking place (Dresing et al. 2014). While cross-field diffusion has traditionally been suggested to transport SEPs across the mean field also other mechanisms, such as largescale drifts (Dalla et al. 2013; Marsh et al. 2013) and propagation along meandering field lines (Giacalone et al. 2000; Laitinen et al. 2013) have received recent attention.

Each of the suggested mechanisms would result in different types of interplanetary transport, which may be seen in the velocity dispersion pattern. However, without taking the diffusion in the interplanetary medium into account in any way, it may be difficult to discern between the different mechanisms. In this work, we derived an estimate for the delay time of SEPs due to the scattering, using a simple model for the onset evolution, and SEP transport simulations. We showed that the estimate improved the VDA fitting results in the case where particles propagate along the Parker spiral field. We tested the model by analyzing a solar event that could be assumed to be magnetically well connected from the Sun to Earth and found that the correction brought both the injection time and path length to be consistent with the expected solar and interplanetary conditions. A comparison of our model results against multi-spacecraft observations, as well as events modeled with cross-field diffusion, drifts, and meandering fieldlines, may bring more light into the mechanism behind the efficient spreading of SEPs in the inner heliosphere.

\section{CONCLUSIONS}

In this work, we have studied the validity of the velocity dispersion method in estimating the injection times of SEPs by using simulations of energetic protons. We find that the typical method of determining the onset time as time when the intensity of the SEPs exceeds the pre-event background in statistically significant amount can lead to significant errors when estimating the solar injection time and path length with the VDA method. It is important to note that a reasonable path length does not imply a good estimate for the injection time.

We studied the use of the intensity increase timescale, $\tau_{I}$ to improve the VDA estimate. The gradual increase of the SEP intensities in time is caused by the scattering of the SEPs in the interplanetary space, and we find a relation between $\tau_{I}$, the diffusion timescale $\tau_{D}$ and the delay of the SEPs with respect to the scatter-free propagation time of the particles from the Sun to the Earth. Using this relation, we showed that the injection time estimate given by the VDA can be improved in cases of magnetically well-connected SEP events.

We conclude that the injection times and path lengths obtained by using the VDA method should be used with care. The interplanetary scattering of particles does delay the arrival of the first-observed SEPs. This delay is likely to cause errors in injection analysis based on the observed SEP onset times particularly if the pre-event particle intensities are high compared to the event maximum intensity. While our analysis was performed using energetic protons, our results are valid for any particle species that are affected by interplanetary scattering, including heavy ions and electrons.

We acknowledge support from the UK Science and Technology Facilities Council (STFC; grants ST/J001341/1 and ST/M00760X/1). The CME catalog is generated and maintained at the CDAW Data Center by NASA and The Catholic University of America in cooperation with the Naval Research Laboratory. SOHO is a project of international cooperation between ESA and NASA.

\section{REFERENCES}

Agueda, N., Lario, D., Vainio, R., et al. 2009, A\&A, 507, 981

Aschwanden, M. J. 2012, SSRv, 171, 3

Cane, H. V., Richardson, I. G., \& von Rosenvinge, T. T. 2010, JGRA, 115 , A08101

Dalla, S., Marsh, M. S., Kelly, J., \& Laitinen, T. 2013, JGRA, 118, 5979

Dresing, N., Gómez-Herrero, R., Heber, B., et al. 2014, A\&A, 567, A27

Dresing, N., Gómez-Herrero, R., Klassen, A., et al. 2012, SoPh, 281, 281

Dröge, W. 2000, ApJ, 537, 1073

Dröge, W. 2003, ApJ, 589, 1027 
Dröge, W., Kartavykh, Y. Y., Klecker, B., \& Kovaltsov, G. A. 2010, ApJ, 709, 912

Effenberger, F., \& Litvinenko, Y. E. 2014, ApJ, 783, 15

Fisk, L. A., \& Axford, W. I. 1969, SoPh, 7, 486

Giacalone, J., Jokipii, J. R., \& Mazur, J. E. 2000, ApJL, 532, L75

Gopalswamy, N., Xie, H., Yashiro, S., et al. 2012, SSRv, 171, 23

Gopalswamy, N., Yashiro, S., Michalek, G., et al. 2009, EM\&P, 104, 295

He, H.-Q., Qin, G., \& Zhang, M. 2011, ApJ, 734, 74

Huttunen-Heikinmaa, K., Valtonen, E., \& Laitinen, T. 2005, A\&A, 442, 673

Jokipii, J. R. 1966, ApJ, 146, 480

Kahler, S. 1994, ApJ, 428, 837

Kallenrode, M., \& Wibberenz, G. 1990, ICRC, 5, 229

Kallenrode, M. B. 1993, JGR, 98, 19037

Kocharov, L., Vainio, R., Kovaltsov, G. A., \& Torsti, J. 1998, SoPh, 182, 195

Krucker, S., \& Lin, R. P. 2000, ApJL, 542, L61

Laitinen, T., Dalla, S., \& Marsh, M. S. 2013, ApJL, 773, L29

Laitinen, T., Huttunen-Heikinmaa, K., \& Valtonen, E. 2010, in AIP Conf.

Proc., Vol. 1216, 12th Int. Solar Wind Conf. (Melville, NY: AIP), 249

Laitinen, T., Klein, K.-L., Kocharov, L., et al. 2000, A\&A, 360, 729

Lin, R. P., Potter, D. W., Gurnett, D. A., \& Scarf, F. L. 1981, ApJ, 251, 364
Lintunen, J., \& Vainio, R. 2004, A\&A, 420, 343

Marsh, M. S., Dalla, S., Kelly, J., \& Laitinen, T. 2013, ApJ, 774, 4

Palmer, I. D. 1982, RvGSP, 20, 335

Parker, E. N. 1965, P\&SS, 13, 9

Reames, D. V. 1999, SSRv, 90, 413

Reames, D. V. 2009, ApJ, 706, 844

Reames, D. V., von Rosenvinge, T. T., \& Lin, R. P. 1985, ApJ, 292, 716

Richardson, I. G., von Rosenvinge, T. T., Cane, H. V., et al. 2014, SoPh, 289, 3059

Sáiz, A., Evenson, P., Ruffolo, D., \& Bieber, J. W. 2005, ApJ, 626, 1131

Torsti, J., Kocharov, L. G., Vainio, R., Anttila, A., \& Kovaltsov, G. A. 1996, SoPh, 166, 135

Torsti, J., Anttila, A., Kocharov, L., et al. 1998, GeoRL, 25, 2525

Torsti, J., Riihonen, E., \& Kocharov, L. 2004, ApJL, 600, L83

Torsti, J., Valtonen, E., Lumme, M., et al. 1995, SoPh, 162, 505

Tylka, A. J., et al. 2003, ICRC, 6, 3305

Vainio, R., Valtonen, E., Heber, B., et al. 2013, JSWSC, 3, A12

Valtonen, E., Kecskeméty, K., Kunow, H., et al. 2001, JGR, 106, 10705

Wiedenbeck, M. E., Mason, G. M., Cohen, C. M. S., et al. 2013, ApJ, 762, 54

Zhang, M., Qin, G., \& Rassoul, H. 2009, ApJ, 692, 109 